\title{
Spiking neural P systems with inhibitory rules
}

\author{
Hong Peng a , Bo Li ${ }^{\text {a }}$, Jun Wang ${ }^{\text {b,* }}$, Xiaoxiao Song ${ }^{\mathrm{b}}$, Tao Wang ${ }^{\mathrm{b}}$, Luis Valencia-Cabrera ${ }^{\mathrm{c}}$, \\ Ignacio Pêrez-Hurtado ${ }^{c}$, Agustín Riscos-Núñez ${ }^{c}$, Mario J. Pêrez-Jiménez ${ }^{c}$ \\ a School of Computer and Software Engineering, Xihua University, Chengdu 610039, China \\ ${ }^{\mathrm{b}}$ School of Electrical Engineering and Electronic Information, Xihua University, Chengdu 610039, China \\ ${ }^{\mathrm{c}}$ Research Group of Natural Computing, Department of Computer Science and Artificial Intelligence, Universidad de Sevilla, Sevilla 41012, Spain
}

Keywords:

Membrane computing

Spiking neural P systems

Spiking neural P systems with inhibitory

rules

Inhibitory synapse

\section{A B S T R A C T}

Motivated by the mechanism of inhibitory synapses, a new kind of spiking neural P (SNP) system rules, called inhibitory rules, is introduced in this paper. Based on this, a new variant of SNP systems is proposed, called spiking neural P systems with inhibitory rules (SNP-IR systems). Different from the usual firing rules in SNP systems, the firing condition of an inhibitory rule not only depends on the state of the neuron associated with the rule but also is related to the states of other neurons. Moreover, from the perspective of topological structure, the new variant is shown as a directed graph with inhibitory arcs, and therefore seems to have more powerful control. The computational completeness of SNPIR systems is discussed. In particular, it is proved that SNP-IR systems are Turing universal number accepting/generating devices. Moreover, we obtain a small universal function-computing device for SNP-IR systems consisting of 100 neurons.

\section{Introduction}

Membrane computing is a class of distributed parallel computing systems initiated by Gheorghe Păun [1], abstracted from the structure and functioning of biological cells as well as the cooperation of cell populations in tissues, organs, and biological neural networks [2]. These computing systems are known as $\mathrm{P}$ systems or membrane systems. Inspired by different biological mechanisms or by combining mathematical methods and/or ideas in computer science, a variety of $\mathrm{P}$ systems have been proposed in the past two decades [3-11]. These can be roughly classified as cell-like, tissue-like, and neural-like P systems. It has been proved that many $\mathrm{P}$ systems and variants are Turing complete (equivalent to a Turing machine) and effective (capable of solving NP-hard problems in a feasible time). Moreover, they have been applied to solve real-world problems [12,13], such as in

\footnotetext{
This work was partially supported by the National Natural Science Founda-tion of China (No. 61472328), Research Fund of Sichuan Science and Technology Project (No. 2018JY0083), Chunhui Project Foundation of the Education Depart-ment of China (Nos. Z2016143 and Z2016148), and Research Foundation of the Education Department of Sichuan province (No. 17TD0034), China.

* Corresponding author. E-mail addresses: ph.xhu@hotmail.com (H. Peng),wj.xhu@hotmail.com (J. Wang).
}

machine learning [14-17], image- and signal-processing [18-24], robots [25,26], ecology, and system biology [27-29].

\subsection{Related work}

As one of the main forms of neural-like $\mathrm{P}$ systems, spiking neural P (SNP) systems, as proposed by Ionescu et al. [30], were abstracted from the biological fact that neurons handle and exchange spikes with each other along synapses. From the perspective of topological structure, an SNP system can be expressed as a directed graph, where the neurons are the nodes of the graph and the synapses correspond to the arcs between them. In addition to its topological structure, an SNP system contains two important components: data and firing rules. The data (denoted by a configuration vector) are used to describe the states of neurons, while the rules (spiking and/or forgetting rules) are used to characterize the dynamic behavior of the system. The firing rules have the form $E / a^{c} \rightarrow a^{p}$, where $E$ denotes the regular expression. The semantics of the firing rule $E / a^{c} \rightarrow a^{p}$ can be explained as follows. Suppose that a neuron where the firing rule resides has $n$ spikes. If $a^{n} \in L(E)$ and $n \geq c$, then the rule is enabled and the neuron fires. Note that $L(E)$ denotes the set of languages generated by the regular expression $E$. When the firing rule is applied, $c$ spikes are removed from the neuron (hence $n-c$ spikes remain) and $p$ new spikes are generated. Then the generated $p$ spikes are sent to its consequent neurons. If $p=0$, then the rule is known as a forgetting rule, written as $E / a^{c} \rightarrow \lambda$, where $\lambda$ denotes the empty string. As described above, 
a firing rule $E / a^{c} \rightarrow a^{p}$ in a neuron has a firing condition: $a^{n} \in$ $L(E)$. It is important to point out that the firing condition is only related to the state of the neuron, and does not depend on the states of other neurons. Neurons work in parallel, so SNP systems are distributed parallel computing models. Nondeterminism is an interesting characteristic of SNP systems: when two or more spiking rules in a neuron can be applied at the same time, one is nondeterministically chosen and applied. Moreover, SNP systems can work in three modes: generating, accepting, and computing.

A variety of SNP systems have been proposed. Motivated by the inhibitory and excitatory influence of astrocytes on synapses, Păun [31] and Pan et al. [32], discussed two SNP systems with astrocytes. Pan et al. [33] discussed SNP systems with anti-spikes by introducing anti-spikes that abstract from inhibitory impulses. Inspired by the biological fact that a synapse has one or more chemical channels, Peng et al. [34] presented SNP systems with multiple channels. Considering that the rules are placed in synapses instead of neurons, SNP systems with rules on synapses have been discussed by Song et al. [35], and another spike-consumption strategy was adopted by Peng et al. [36]. Chen et al. [37] investigated an axon P system where nodes were arranged in a linear structure and each only sent spikes to two neighbors. Inspired by the fact that every neuron has a positive or negative charge, SNP systems with polarizations have been discussed [38]. The structural dynamism of biological synapses inspired Cabarle et al. [39] to present an SNP system with scheduled synapses. Considering a new communication strategy among neurons, Pan et al. [40] discussed SNP systems with communication on request. With the limitation that at most one neuron works at each step, several sequential SNP systems were investigated by Ibarra et al. [41] and Zhang et al. [42]. Wang et al. [43] discussed an SNP system in which weights like those in artificial neural networks were introduced on synapses. Dynamic threshold neural P systems and coupled neural P systems were discussed by Peng et al. [44,45]. Moreover, using the thresholds instead of the original regular expression, Zeng et al. [46] proposed SNP systems with thresholds. Note that in SNP systems with thresholds, the firing condition is changed as $n \geq T$. A global clock is usually assumed in SNP systems, so they are synchronized. However, Cavaliere et al. [47] and Song et al. [48] investigated two asynchronous SNP systems. By integrating fuzzy logic in SNP systems, several fuzzy SNP systems have been developed, such as fuzzy reasoning SNP systems [49], weighted fuzzy SNP systems [50], and interval-valued fuzzy SNP systems [51].

Computational properties of variants of SNP systems have been investigated. As function-computing, natural numbergenerating, and language-generating devices, most have been proved to be Turing universal [52-55]. Furthermore, SNP systems have been applied to (theoretically) solve a number of computationally hard problems in a feasible (polynomial or linear) time. Application of SNP systems in some real-world problems, such as fault diagnosis [56-59], image processing [60], and combinatorial optimization [61], has recently received much attention.

\subsection{Motivation}

As mentioned above, data (i.e., states) and rules (firing and forgetting rules) are two important components in SNP systems and their variants. The firing condition $a^{n} \in L(E)$ is only related to the state of the neuron with which the firing rule is associated. Hence whether a neuron fires depends solely on its current state and has nothing to do with the states of other neurons. Therefore, the behavior of each neuron in an SNP system is controlled only by its state (the number of spikes). From this, an interesting idea emerges: can the firing of a neuron be controlled by the states of other neurons? Is there a biological fact that can support this interesting idea?
Two scientific findings in biological nervous systems [62,63] are related to this idea.

(1) Excitatory synapse [64]

The excitatory synapse transfers the excitation of presynaptic to postsynaptic. Once the action potential at the end of presynaptic fiber is reached, the excitatory synapse chemically or electrically passes it to the postsynaptic neurons and produces excitatory postsynaptic potentials. Excitatory postsynaptic potential is a depolarizing potential change that can be summed up by the activity of multiple excitatory synapses, and action potential is generated when it exceeds the threshold.

(2) Inhibitory synapse [65]

Presynaptic excitatory transmission has an inhibitory effect on postsynaptic excitation. When the excitatory (action) potential reaches the tip of the presynaptic fiber, it is transferred chemically or electrically to the postsynaptic neuron, where the inhibitory postsynaptic potential is produced. Inhibitory postsynaptic potential reduces the depolarization of the excitatory postsynaptic potential and thus inhibits action potential because of the short-circuit effect caused by hyperpolarization and increased ion permeability.

In SNP systems, spikes received by a neuron from other neurons are accumulated to update the state of the neuron, and if the firing condition is satisfied, then some new spikes will be generated. Therefore, the functioning of an excitatory synapse is basically consistent with the firing mechanism in SNP systems and their variants. However, the functioning of an inhibitory synapse is not reflected in these systems. The main motivation of this paper is to develop an inhibitory rule based on the functioning of inhibitory synapses and then to propose a new model of SNP systems, SNP systems with inhibitory rules (SNP-IRs).

An inhibitory rule has the form $\left(E_{\text {en }}, \overline{E_{i n}}\right) / a^{c} \rightarrow a^{p}$, where $E_{\text {en }}$ is called enable regular expression, while $E_{\text {in }}$ is called inhibitory regular expression. The semantics of inhibitory rules will be explained in detail later. The new firing condition can be written as $a^{n_{1}} \in L\left(E_{\text {en }}\right) \wedge a^{n_{2}} \notin L\left(E_{\text {in }}\right)$, where $n_{1}$ is the number of spikes in the neuron where the inhibitory rule resides, and $n_{2}$ is the number of spikes in its preceding neuron (called an inhibitory neuron). Therefore, the firing condition indicates that the firing of an inhibitory rule in a neuron not only depends on its state but also is related to the state of other (inhibitor) neurons, which corresponds to the functioning of an inhibitory synapse.

The new variant differs from SNP systems in the following ways.

(1) The new variant introduces an inhibitory rule, inspired by the functioning of an inhibitory synapse.

(2) In the new variant, the firing of rules depends on both a neuron's state and those of other (inhibitor) neurons. However, the firing of rules in an SNP system is only related to a neuron's state. Therefore, the new variant seems to have more powerful control than an SNP system.

(3) Topologically, the new variant can be expressed as a directed graph with inhibitory arcs, which do not appear in standard SNP systems.

In summary, the novelty of the work is to abstract an inhibitory rule inspired by the functioning of an inhibitory synapse and to propose SNP systems with inhibitory rules.

The remainder of this paper is arranged as follows. Section 2 defines SNP-IR systems and provides an illustrative example. Section 3 studies the computational completeness of SNP-IR systems as number-generating/accepting and function-computing devices. Conclusions are drawn and further work is suggested in Section 4. 


\section{SNP-IR systems}

To define SNP-IR systems more clearly, some notions and notations related to both SNP systems and formal language theory are briefly reviewed. Further details can be found in $[2,66]$.

Let $\Sigma$ be an alphabet. The set of all of the finite strings over $\Sigma$ is denoted by $\Sigma^{*}$, the empty string is written as $\lambda$, and the set of all of the nonempty strings over $\Sigma$ is denoted by $\Sigma^{+}$. Usually, if $\Sigma=\{a\}$, then $\{a\}^{*}$ and $\{a\}^{+}$can be simply written as $a^{*}$ and $a^{+}$, respectively.

A regular expression over $\Sigma$ is defined recursively: (i) $\lambda$ and every $a \in \Sigma$ are regular expressions; (ii) if $E_{1}, E_{2}$ are two regular expressions over $\Sigma$, then $\left(E_{1}\right)\left(E_{2}\right),\left(E_{1}\right) \cup\left(E_{2}\right)$, and $\left(E_{1}\right)^{+}$are regular expressions over $\Sigma$; (iii) nothing else is a regular expression over $\Sigma$. A language $L(E)$ can be associated with regular expression $E$ over $\Sigma$ as follows: (i) $L(\lambda)=\lambda$ and $\forall a \in \Sigma, L(a)=\{a\}$; (ii) for any two regular expressions $E_{1}, E_{2}, L\left(\left(E_{1}\right) \cup\left(E_{2}\right)\right)=L\left(E_{1}\right) \cup L\left(E_{2}\right)$, $L\left(\left(E_{1}\right)\left(E_{2}\right)\right)=L\left(E_{1}\right) L\left(E_{2}\right)$, and $L\left(\left(E_{1}\right)^{+}\right)=\left(L\left(E_{1}\right)\right)^{+}$.

\subsection{Definition}

Definition 1. An SNP-IR system of degree $m \geq 1$ is a tuple:

$\Pi=\left(0, \sigma_{1}, \sigma_{2}, \ldots, \sigma_{m}\right.$, syn, in, out $)$,

where:

(1) $O=\{a\}$ denotes a singleton alphabet ( $a$ is known as the spike);

(2) $\sigma_{1}, \sigma_{2}, \ldots, \sigma_{m}$ are $m$ neurons, denoted by $\sigma_{i}=\left(n_{i}, R_{i}\right), 1 \leq$ $i \leq m$, where:

(a) $n_{i} \geq 0$ denotes the number of spikes stored initially in neuron $\sigma_{i}$;

(b) $R_{i}$ denotes the finite set of rules of two types:

(i) usual firing rules, of the form $E / a^{c} \rightarrow a^{p}$;

(ii) inhibitory rules, of the form $\left(E_{e n}, \overline{E_{i n(i, j)}}\right) / a^{c} \rightarrow$ $a^{p}$

where $E, E_{\text {en }}, E_{i n(i, j)}$ are the regular expressions over 0 , and $c \geq 1, p \geq 0$, and $c \geq p$;

(4) syn $=\{(i, j)\} \subseteq\{1,2, \ldots, m\} \times\{1,2, \ldots, m\}$ with $(i, i) \notin$ syn $\forall 1 \leq i \leq m$ (synapse connections);

(5) in, out $\in\{1,2, \ldots, m\}$, respectively, distinguish input and output neurons.

From the perspective of a topological structure, the SNP-IR system is presented as a directed graph with inhibitory arcs, where $m$ neurons are the nodes of the graph, and the synapses correspond to the arcs between the nodes. Specifically, if the system $\Pi$ works in generative mode, then input neuron $\sigma_{i n}$ is removed from the system; by contrast, in accepting mode, output neuron $\sigma_{\text {out }}$ is omitted.

As usual, the state of a neuron at time $t$ is denoted by the number of spikes, while the state of the whole system at that time is characterized by the configuration vector $C_{t}=\left(n_{1}(t), n_{2}(t), \ldots\right.$, $n_{m}(t)$ ), where $c_{i}(t)$ is the number of spikes in neuron $\sigma_{i}, 1 \leq$ $i \leq m$. Thus the initial configuration is $C_{0}=\left(n_{1}, n_{2}, \ldots, n_{m}\right)$. As a result, a transition from one configuration to another can be defined. Usually, such a sequence of transitions starting from the initial configuration is known as a computation. If a configuration where no rule can be applied is attained in a computation, then it halts.

There are two types of rules in SNP-IR systems: usual firing rules and inhibitory rules. As in SNP systems, usual firing rules have the form $E / a^{c} \rightarrow a^{p}$. Suppose that a firing rule $E / a^{c} \rightarrow a^{p}$ is in neuron $\sigma_{i}$, and the neuron has $n_{i}(t)$ spikes. If $a^{n_{i}(t)} \in L(E)$ and

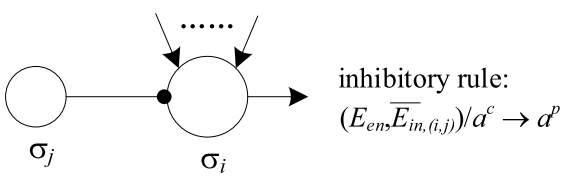

(a)

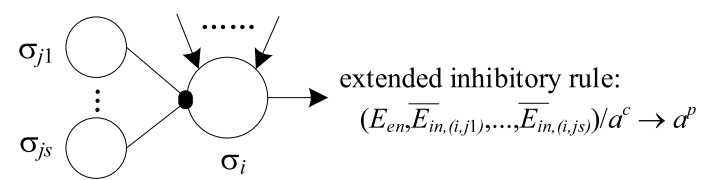

(b)

Fig. 1. Rules: (a) inhibitory rule, and (b) extended inhibitory rule.

$n_{i}(t) \geq c$, then the neuron fires and $c$ spikes are removed from the neuron $\left(n_{i}(t)-c\right.$ spikes are retained), and then $p$ new spikes are generated. The generated spikes are sent to its succeeding neurons. If $q=0$ in the rule, then it can written as $E / a^{c} \rightarrow$ $\lambda$, which is known as a forgetting rule. If the forgetting rule is applied, then $c$ spikes are removed but no spike is generated.

Inhibitory rules are of the form $\left(E_{e n}, \overline{E_{i n,(i, j)}}\right) / a^{c} \rightarrow a^{p}$, as shown in Fig. 1(a). Each inhibitory rule has two regular expressions to control the firing of a neuron: an enable regular expression $E_{e n}$ and an inhibitory regular expression $E_{i n,(i, j)}$. Suppose that the inhibitory rule $\left(E_{e n}, \overline{E_{i n,(i, j)}}\right) / a^{c} \rightarrow a^{p}$ is associated with neuron $\sigma_{i}$. The enable regular expression $E_{e n}$ is only related to neuron $\sigma_{i}$, and its firing condition is $a^{n_{i}(t)} \in L\left(E_{e n}\right)$. For the inhibitory regular expression $E_{i n,(i, j)}$, its firing condition is $a^{n_{j}(t)} \notin L\left(E_{i n,(i, j)}\right)$, since it is only related to the state of neuron $\sigma_{j}$. Thus the firing condition of neuron $\sigma_{i}$ can be denoted by a Boolean expression, $a^{n_{i}(t)} \in L\left(E_{e n}\right) \wedge$ $a^{n_{j}(t)} \notin L\left(E_{i n,(i, j)}\right)$. Note that in $E_{i n,(i, j)}$, the subscript $(i, j)$ indicates that there is an arc, called an inhibitory arc, between neurons $\sigma_{i}$ and $\sigma_{j}$. An inhibitory arc that corresponds to an inhibitory synapse is denoted by an arc with a solid circle, as shown in Fig. 1(a), and neuron $\sigma_{j}$ is therefore called the inhibitory neuron of $\sigma_{i}$. A directed arc with an arrow corresponds to an excitatory synapse. For neurons $\sigma_{i}$ and $\sigma_{j}$, the following assumptions are given:

(1) If neurons $\sigma_{i}$ and $\sigma_{j}$ have an inhibitory arc, then there is no usual arc between them.

(2) If neuron $\sigma_{j}$ is an inhibitory neuron of neuron $\sigma_{i}$, then neuron $\sigma_{j}$ cannot send a spike to neuron $\sigma_{i}$. Moreover, the number of spikes in neuron $\sigma_{j}$ cannot be changed even if some inhibitory rule in neuron $\sigma_{i}$ is applied. Therefore, for neuron $\sigma_{i}$, neuron $\sigma_{j}$ only plays the role of controlling the firing.

A neuron may have more than one inhibitory neuron; for example, neuron $\sigma_{i}$ in Fig. 1 (b) has $s$ inhibitory neurons, $\sigma_{j 1}, \sigma_{j 1}, \ldots$, $\sigma_{j s}$. The inhibitory rule of the form $\left(E_{e n}, \overline{E_{i n,(i, j 1)}}, \ldots, \overline{E_{i n,(i, j s)}}\right) / a^{c} \rightarrow$ $a^{p}$ is called an extended inhibitory rule, whose firing condition can be denoted by $a^{n_{i}(t)} \in L\left(E_{e n}\right) \wedge a^{n_{j 1}(t)} \notin L\left(E_{i n,(i, j 1)}\right) \wedge \cdots \wedge a^{n_{j s}(t)} \notin$ $L\left(E_{i n,(i, j s)}\right)$.

Based on the above firing mechanism, the state of neuron $\sigma_{i}$ at time $t+1$ can be computed as

$n_{i}(t+1)= \begin{cases}n_{i}(t)-c+n, & \text { if rule is used; } \\ n_{i}(t)+n, & \text { otherwise }\end{cases}$

where $n$ is the number of spikes retrieved from other neurons. Note that whether a firing rule or inhibitory rule is applied, $c$ spikes are removed from neuron $\sigma_{i}$.

In every computing step, if a neuron $\sigma_{i}$ can use one of its rules, then a rule from $R_{i}$ must be used. The firing conditions of two rules in a neuron may be satisfied simultaneously, such as 


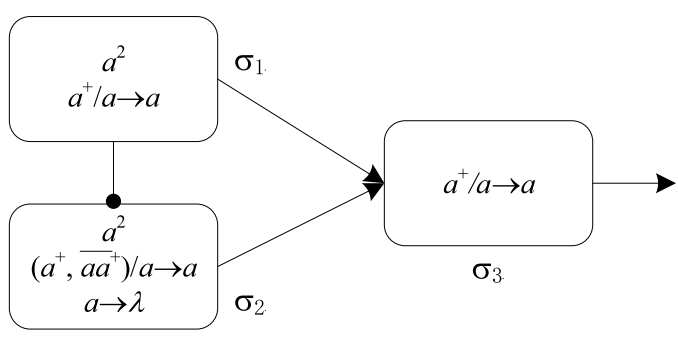

Fig. 2. An illustrative example.

two usual firing rules, two inhibitory rules, or a usual firing rule and an inhibitory rule. In either case, one of these enabled rules will be chosen nondeterministically. Consequently, the rules are applied sequentially in every neuron, whereas neurons work in parallel.

Any computing can correspond to a spike train consisting of zeros and ones, which describe the behavior of the output neuron: write 1 when the output neuron excites the spikes, and write 0 when the output neuron fires. Hence the number of steps between the first two spikes excited by the output neuron is regarded as the computational result. Let $N_{2}(\Pi)$ be the set of numbers computed by $\Pi . N_{2} S N P_{m}^{n}$ denotes the families of all sets $N_{2}(\Pi)$ computed by an SNP-IR system, consisting of at most $m$ neurons and at most $n$ rules in every neuron. Note that if one of $m$ and $n$ is not limited, then the symbol "*" is used to substitute it.

We can execute the SNP-IR system $\Pi$ under the accepting mode: the spikes are received by an input neuron from the environment, and the output neuron is ignored. The system starts by importing the spike train from the environment, and then it stores the number $n$ to a specified neuron in the form of $2 n$ spikes. When the system halts, $n$ is known as the number accepted by it. Denote by $N_{a c c}(\Pi)$ the set of numbers accepted by the system $\Pi$, in which the subscript acc identifies that the system works in the accepting mode. $N_{a c c} S N P_{m}^{n}$ denotes the family of all sets $N_{a c c}(\Pi)$ accepted by an SNP-IR system consisting of at most $m$ neurons and at most $n$ rules in every neuron.

\subsection{An illustrative example}

An example that can generate a string of zeros and ones is provided to clarify the working mechanism of SNP-IR systems. Suppose an SNP-IR system has three neurons, $\sigma_{1}, \sigma_{2}$, and $\sigma_{3}$, as shown in Fig. 2. Note that out $=3$, i.e., neuron $\sigma_{3}$ is an output neuron.

Initially, neurons $\sigma_{1}$ and $\sigma_{2}$ each have two spikes, while neuron $\sigma_{3}$ has no spike. Therefore, the initial configuration is $C_{0}=$ $(2,2,0)$.

At time 1 , since neuron $\sigma_{1}$ has two spikes, rule $a^{+} / a \rightarrow a$ can be applied. After applying the rule, a spike will be consumed and a new spike will be generated and sent to neuron $\sigma_{3}$. Note that even if neuron $\sigma_{2}$ has two spikes that satisfy $a^{2} \in L\left(a^{+}\right)$, two spikes in neuron $\sigma_{1}$ can inhibit the rule $\left(a^{+}, \overline{a a^{+}}\right) / a \rightarrow a$ in neuron $\sigma_{2}$ because $a^{2} \in L\left(a a^{+}\right)$, so no rule is enabled in neuron $\sigma_{2}$. Similarly, no rule is enabled in neuron $\sigma_{3}$. Therefore, $C_{1}=(1,2,1)$.

At time 2, with a spike in neuron $\sigma_{3}$, rule $a^{+} / a \rightarrow a$ is applied to send a spike to the environment. With a spike in neuron $\sigma_{1}$, rule $a^{+} / a \rightarrow a$ is applied to send a spike to neuron $\sigma_{3}$. Since neuron $\sigma_{2}$ has two spikes and neuron $\sigma_{1}$ has one spike, the firing condition of rule $\left(a^{+}, \overline{a a^{+}}\right) / a \rightarrow a$ is satisfied, i.e., $a^{2} \in L\left(a^{+}\right) \wedge$ $a^{2} \notin L\left(a a^{+}\right)$, hence the rule is applied to send a spike to neuron $\sigma_{3}$. After neurons $\sigma_{1}$ and $\sigma_{2}$ fire, neuron $\sigma_{3}$ will receive two spikes. Thus $C_{2}=(0,1,2)$.
At time 3, due to two spikes in neuron $\sigma_{3}$, rule $a^{+} / a \rightarrow a$ is applied to send a spike to the environment. Since neuron $\sigma_{2}$ has a spike and neuron $\sigma_{1}$ has no spike, rule $\left(a^{+}, \overline{a a^{+}}\right) / a \rightarrow a$ and rule $a \rightarrow \lambda$ are enabled simultaneously. Therefore, one of the two rules is nondeterministically chosen and applied, and the following two cases are considered:

(1) If rule $\left(a^{+}, \overline{a a^{+}}\right) / a \rightarrow a$ is applied, then neuron $\sigma_{2}$ sends a spike to neuron $\sigma_{3}$. Thus $C_{3}=(0,0,2)$. At time 4 , rule $a^{+} / a \rightarrow a$ in neuron $\sigma_{3}$ is applied to send a spike to the environment. Then neuron $\sigma_{3}$ again sends a spike to the environment. Therefore, the spike train generated by the system is " 01111 ".

(2) If rule $a \rightarrow \lambda$ is applied, then the spike in neuron $\sigma_{2}$ is consumed by rule $a \rightarrow \lambda$. Thus $C_{3}=(0,0,1)$. At time 4 , rule $a^{+} / a \rightarrow a$ in neuron $\sigma_{3}$ is applied to send a spike to the environment. Therefore, the spike train generated by the system is " 0111 ".

\section{Computation completeness}

In this section, we will discuss the universality of SNP-IR systems as devices for number generating, number accepting, and function computing. The universality of SNP-IR systems will be proven by the simulation of register machines, by which all recursively enumerable sets of numbers can be generated/accepted by SNP-IR systems. Moreover, a small universal function-computing device can be constructed.

A register machine is given as a tuple, $M=\left(m, H, l_{0}, l_{h}, I\right)$, where $m$ indicates the number of registers, $H$ is the set of instruction labels, $l_{0}$ is the start label, $l_{h}$ is the halting instruction label, and $I$ is the set of instructions. Each instruction in $I$ is associated with a label in $H$. $I$ has three types of instructions:

(1) $l_{i}:\left(A D D(r), l_{j}, l_{k}\right)$ (add 1 to register $r$ and then nondeterministically move to one of the instructions with labels $l_{j}$, $\left.l_{k}\right)$.

(2) $l_{i}:\left(\operatorname{SUB}(r), l_{j}, l_{k}\right)$ (if register $r$ is nonzero, then decrement it by 1 and move to the instruction with label $l_{j}$; otherwise, move to the instruction with label $l_{k}$ ).

(3) $l_{h}:$ HALT (halting instruction).

\subsection{SNP-IR systems as number generating devices}

A number $n$ can be computed by a register machine working in the generating mode. Starting from the instruction with label $l_{0}$ and with all of the empty registers, the machine constantly applies instructions as distinguished by labels until it halts; at this moment, the number contained in the first register is known as the result computed by $M$. As we know, the family NRE can be characterized by register machines.

Theorem 1. $\mathrm{N}_{2} S N P_{*}^{2}=N R E$

Proof. Because $N_{2} S N P_{*}^{2} \subseteq N R E$ is straightforward, it is only necessary to prove the inclusion $N R E \subseteq N_{2} S N P_{*}^{2}$. Hence a register machine $M=\left(m, H, l_{0}, l_{h}, I\right)$ working in the generative mode is considered. In general, all of the registers different from register 1 are assumed to be empty in the halting configuration, and during the computation, register 1 is never decremented.

To simulate register machine $M$, an SNP-IR system $\Pi_{1}$ is designed, which contains modules of three types: an ADD module to simulate the ADD instruction (Fig. 3), a SUB module to simulate the SUB instruction (Fig. 4), and a FIN module to output the computational result (Fig. 5). 


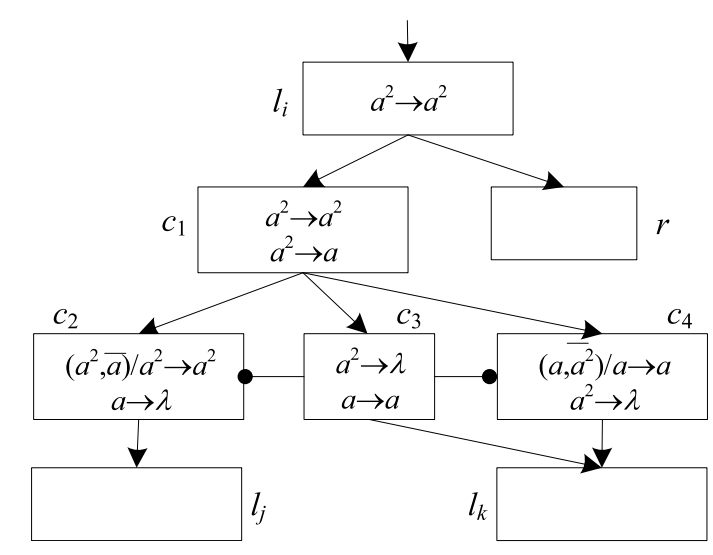

Fig. 3. ADD module, simulating the ADD instruction $l_{i}:\left(A D D(r), l_{j}, l_{k}\right)$.

Assume that every register $r$ is associated with a neuron $\sigma_{r}$. We can code the number in register $r$ : if register $r$ stores the number $n \geq 0$, then neuron $\sigma_{r}$ has $2 n$ spikes. We associate a neuron $\sigma_{l}$ with every instruction $l$ in $H$, and we introduce some auxiliary neurons to these modules. Assume that each auxiliary neuron initially has no spike, and each neuron $\sigma_{l_{i}}$ that is associated with $l_{i}$ contains two spikes. Due to two spikes in neuron $\sigma_{l_{i}}$, the system $\Pi_{1}$ starts the simulation of the instruction $l_{i}:\left(O P(r), l_{j}, l_{k}\right)(O P$ identifies one of the operations ADD and SUB). Starting from the activation of neuron $l_{i}$, the simulation deals with neuron $\sigma_{r}$ as identified by OP, and two spikes are introduced in one of the neurons $\sigma_{l_{j}}$ and $\sigma_{l_{k}}$. The computation in $M$ is continually simulated until neuron $\sigma_{l}$ fires. During the computation, the spikes are sent into the environment twice, at times $t_{1}$ and $t_{2}$, and the computational result is the value $t_{2}-t_{1}$ associated with the number contained in register 1 .

To illustrate that the register machine $M$ is correctly simulated by the system $\Pi_{1}$, we will discuss how ADD and SUB modules simulate ADD and SUB instructions, and how the computational result is exported by the FIN module.

(1) ADD module (Fig. 3) - simulating an ADD instruction $l_{i}$ : $\left(A D D(r), l_{j}, l_{k}\right)$.

The system $\Pi_{1}$ starts from the simulation of instruction $l_{0}$, which is an ADD instruction. Assume that an ADD instruction $l_{i}:\left(A D D(r), l_{j}, l_{k}\right)$ is simulated at time $t$. At this moment, neuron $\sigma_{l_{i}}$ has two spikes. Hence, rule $a^{2} \rightarrow a^{2}$ is used to send two spikes to neurons $\sigma_{c_{1}}$ and $\sigma_{r}$. Neuron $\sigma_{r}$ receives two spikes, meaning that register $r$ is incremented by 1 . At time $t+1$, with two spikes in neuron $\sigma_{c_{1}}$, rules $a^{2} \rightarrow a^{2}$ and $a^{2} \rightarrow a$ can be applied simultaneously. Therefore, one of the two rules in neuron $\sigma_{c_{1}}$ is chosen nondeterministically. There exist the following two cases:

(i) At time $t+1$, if rule $a^{2} \rightarrow a^{2}$ is used, then neuron $\sigma_{c_{1}}$ sends two spikes to each of neurons $\sigma_{c_{2}}, \sigma_{c_{3}}$, and $\sigma_{c_{4}}$. At time $t+2$, since neuron $\sigma_{c_{3}}$ has two spikes, rule $\left(a^{2}, \bar{a}\right) \rightarrow a^{2}$ in neuron $\sigma_{c_{2}}$ is enabled, but rule $\left(a, \overline{a^{2}}\right) \rightarrow a$ in neuron $\sigma_{c_{4}}$ is inhibited. At time $t+3$, neuron $\sigma_{l_{j}}$ receives two spikes from neuron $\sigma_{c_{2}}$, meaning that the system $\Pi_{1}$ starts to simulate instruction $l_{j}$. Simultaneously, two spikes in neurons $\sigma_{c_{3}}$ and $\sigma_{c_{4}}$ are consumed by rule $a^{2} \rightarrow \lambda$.

(ii) At time $t+1$, if rule $a^{2} \rightarrow a$ is used, then neuron $\sigma_{c_{1}}$ sends a spike to neurons $\sigma_{c_{2}}, \sigma_{c_{3}}$, and $\sigma_{c_{4}}$. At time $t+2$, since neuron $\sigma_{c_{3}}$ has a spike, rule $\left(a, \overline{a^{2}}\right) \rightarrow a$ in neuron $\sigma_{c_{4}}$ is enabled, but rule $\left(a^{2}, \bar{a}\right) \rightarrow a^{2}$ in neuron $\sigma_{c_{2}}$ is inhibited. At this time, rule $a \rightarrow a$ in neuron $\sigma_{c_{3}}$ is enabled. At time $t+3$, neuron $\sigma_{l_{k}}$ receives two spikes in total from neurons $\sigma_{c_{3}}$ and $\sigma_{c_{4}}$,

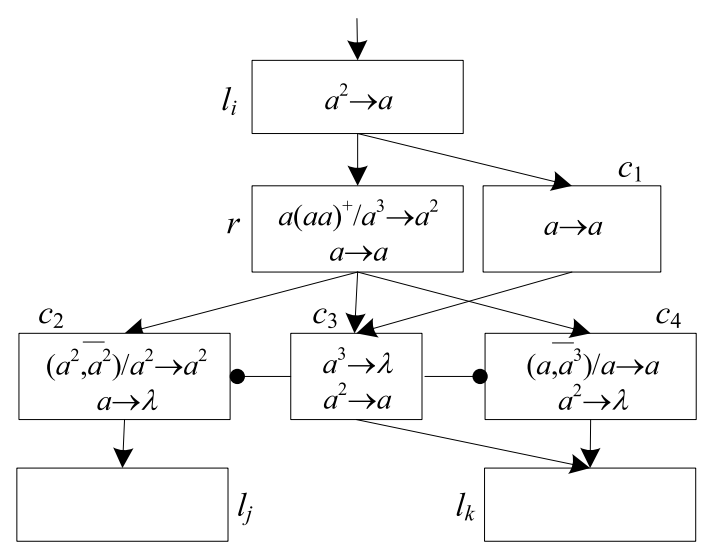

Fig. 4. SUB module, simulating the SUB instruction $l_{i}:\left(\operatorname{SUB}(r), l_{j}, l_{k}\right)$.

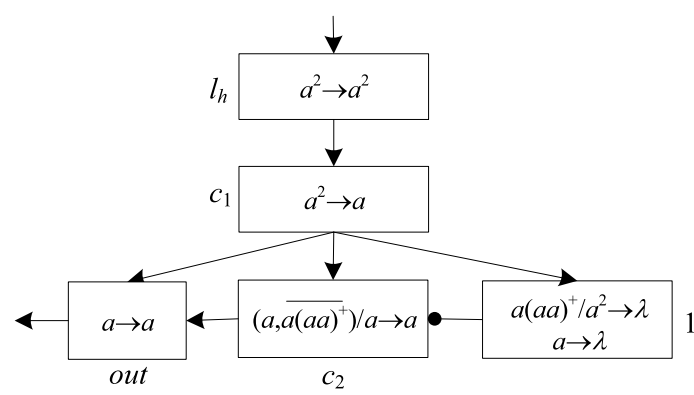

Fig. 5. FIN module.

meaning that the system $\Pi_{1}$ starts to simulate instruction $l_{k}$. Simultaneously, the spike in neuron $\sigma_{c_{2}}$ is consumed by rule $a \rightarrow \lambda$

Therefore, the ADD module can correctly simulate the ADD instruction: when neuron $\sigma_{l_{i}}$ receives two spikes, the number of spikes in neuron $\sigma_{r}$ is increased by two, and one of the neurons $\sigma_{l_{j}}$ and $\sigma_{l_{k}}$ is nondeterministically chosen.

(2) SUB module (Fig. 4) - simulating a SUB instruction $l_{i}$ : $\left(\operatorname{SUB}(r), l_{j}, l_{k}\right)$.

Suppose that a SUB instruction $l_{i}:\left(\operatorname{SUB}(r), l_{j}, l_{k}\right)$ is simulated at time $t$. At this moment, neuron $\sigma_{l_{i}}$ has two spikes, hence its rule $a^{2} \rightarrow a$ is used to send a spike to neurons $\sigma_{r}$ and $\sigma_{c_{1}}$. At time $t+1$, rule $a \rightarrow a$ in neuron $\sigma_{c_{1}}$ is used to send a spike to neuron $\sigma_{c_{3}}$. Based on the number of spikes in neuron $\sigma_{r}$, there exist the following two cases:

(i) At time $t+1$, if neuron $\sigma_{r}$ contains $2 n+1(\geq 3)$ spikes (because the number in register $r$ is $n$ ), then rule $a(a a)^{+} / a^{3} \rightarrow$ $a^{2}$ is used to send two spikes to neurons $\sigma_{c_{2}}, \sigma_{c_{3}}$, and $\sigma_{c_{4}}$. At time $t+2$, since neuron $\sigma_{c_{3}}$ has three spikes, rule $\left(a^{2}, \overline{a^{2}}\right) / a^{2} \rightarrow a^{2}$ in neuron $\sigma_{c_{2}}$ is applied to send two spikes to neuron $\sigma_{l_{j}}$, but rule $\left(a, \overline{a^{3}}\right) / a \rightarrow a$ in neuron $\sigma_{c_{4}}$ is inhibited. Moreover, three spikes in neuron $\sigma_{c_{3}}$ and two spikes in neuron $\sigma_{c_{4}}$ are consumed. Hence neuron $\sigma_{l_{j}}$ has two spikes, meaning that the system $\Pi_{1}$ starts to simulate instruction $l_{j}$.

(ii) At time $t+1$, if neuron $c_{r}$ has only a spike (since the number in register $r$ is still zero), rule $a \rightarrow a$ is applied and a spike is sent to each of neurons $\sigma_{c_{2}}, \sigma_{c_{3}}$, and $\sigma_{c_{4}}$. At time $t+2$, since neuron $\sigma_{c_{3}}$ has two spikes, rule $\left(a, \overline{a^{3}}\right) / a \rightarrow a$ in neuron $\sigma_{c_{4}}$ is applied to send a spike to neuron $\sigma_{l_{k}}$, but rule $\left(a^{2}, \overline{a^{2}}\right) / a^{2} \rightarrow a^{2}$ in neuron $\sigma_{c_{2}}$ is inhibited. Moreover, 
due to two spikes in neuron $\sigma_{c_{3}}, a^{2} \rightarrow a$ is applied to send a spike to neuron $\sigma_{l_{k}}$, and a spike in neuron $\sigma_{c_{2}}$ is consumed by rule $a \rightarrow \lambda$. Thus neuron $\sigma_{l_{k}}$ has two spikes, meaning that the system $\Pi_{1}$ starts to simulate instruction $l_{j}$.

Consequently, the SUB instruction can be correctly simulated by the SUB module: the system starts from neuron $\sigma_{l_{i}}$ receiving two spikes, and ends with the sending of two spikes to neuron $\sigma_{l_{j}}$ (if the number in register $r$ is greater than 0 ), or sending two spikes to neuron $\sigma_{l_{k}}$ (if the number in register $r$ is 0 ).

(3) FIN module (Fig. 5) - outputting the computation result.

Assume that neuron $\sigma_{l_{h}}$ has two spikes at time $t$, meaning that $M$ halts, and neuron $\sigma_{1}$ contains $2 n$ spikes (i.e., register 1 contains the number $n$ ). Due to two spikes in neuron $\sigma_{l_{h}}$, rule $a^{2} \rightarrow a^{2}$ is applied to send two spikes to neuron $\sigma_{c_{1}}$. At time $t+1$, neuron $\sigma_{c_{1}}$ sends a spike to neurons $\sigma_{c_{2}}, \sigma_{\text {out }}$, and $\sigma_{1}$. At time $t+2$, due to a spike in neuron $\sigma_{\text {out }}$, rule $a \rightarrow a$ is applied to send the first spike into the environment. Note that neuron $\sigma_{1}$ is an inhibitory neuron of neuron $\sigma_{c_{2}}$. Since neuron $\sigma_{1}$ has $(2 n+1) \geq 3$ spikes, rule $\left(a, \overline{a(a a)^{+}}\right) / a \rightarrow a$ in neuron $\sigma_{c_{2}}$ is inhibited. Moreover, two spikes in neuron $\sigma_{1}$ are consumed by rule $a(a a)^{+} / a^{2} \rightarrow \lambda$. The process is repeated until neuron $\sigma_{1}$ has only a spike.

At time $t+n+1$, since neuron $\sigma_{1}$ has only a spike, rule $\left(a, \overline{a(a a)^{+}}\right) / a \rightarrow a$ in neuron $\sigma_{c_{2}}$ is applied to send a spike to neuron $\sigma_{\text {out }}$. At this time, the spike in neuron $\sigma_{1}$ is removed by rule $a \rightarrow \lambda$. At time $t+n+2$, neuron $\sigma_{\text {out }}$ sends a spike to the environment. Consequently, the interval between the two spikes sent to the environment by the system is $(t+n+2)-(t+2)=n$, which indicates exactly the number in register 1 when $M$ halts.

From the discussion above, the system $\Pi_{1}$ correctly simulates the register machine $M$ working in generating mode, in which each neuron contains two rules at most. Therefore, the theorem holds.

\subsection{SNP-IR systems as number accepting devices}

The number $n$ can usually be accepted by a register machine working in the accepting mode as follows. The machine first reads a spike train that codes a number from the environment and stores it in the first register, and all of the registers are assumed to be empty. Then, starting from the instruction with label $l_{0}$, the machine continually uses the instructions as identified by labels. When the halting instruction is reached, the number is said to be accepted by $M$.

Theorem 2. $N_{a c c} S N P_{*}^{2}=N R E$

Proof. An SNP-IR system $\Pi_{2}$ working in accepting mode is designed to simulate the deterministic register machine $M=$ ( $m, H, l_{0}, l_{h}, I$ ). The proof will be described by a modification of the proof of Theorem 1 . The system $\Pi_{2}$ contains modules of three types: a deterministic ADD module, a SUB module, and an INPUT module.

Fig. 6 shows the INPUT module. Neuron $\sigma_{i n}$ is used to read spike train $10^{n-1} 1$ from the environment, where the interval between the two spikes in the spike train is $(n+1)-1=n$, which is the number to be accepted.

Suppose that at time $t$, neuron $\sigma_{\text {in }}$ reads the first spike from the environment. At time $t+1$, rule $a \rightarrow a$ in neuron $\sigma_{\text {in }}$ is applied to send a spike to neurons $\sigma_{c_{1}}, \sigma_{c_{2}}$, and $\sigma_{c_{3}}$. Note that neuron $\sigma_{c_{3}}$ is the inhibitory neuron of neurons $\sigma_{c_{2}}$ and $\sigma_{c_{1}}$. At time $t+2$, because neuron $\sigma_{c_{2}}$ contains only a spike, rule $\left(a, \overline{a^{2}}\right) / a \rightarrow a$ in neurons $\sigma_{c_{2}}$ and $\sigma_{c_{1}}$ is enabled. Neurons $\sigma_{c_{2}}$ and $\sigma_{c_{1}}$ send a spike to neuron $\sigma_{1}$, and they exchange a spike with each other. As a result, neuron $\sigma_{1}$ receives two spikes, and a spike is still retained in each of neurons $\sigma_{c_{2}}$ and $\sigma_{c_{1}}$. The process is repeated until the

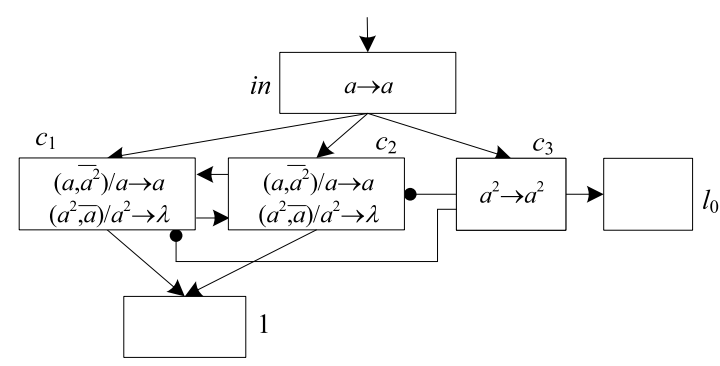

Fig. 6. INPUT module.

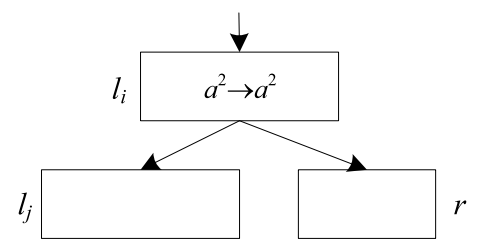

Fig. 7. ADD module, simulating $l_{i}:\left(A D D(r), l_{j}\right)$.

second spike arrives at neurons $\sigma_{c_{1}}, \sigma_{c_{2}}$, and $\sigma_{c_{3}}$. During each step, neurons $\sigma_{c_{2}}$ and $\sigma_{c_{1}}$ exchange a spike with each other, and two spikes are added to neuron $\sigma_{1}$.

At time $t+n-1$, neuron $\sigma_{\text {in }}$ reads the second spike from the environment. At time $t+n$, neuron $\sigma_{\text {in }}$ sends a spike to neurons $\sigma_{c_{1}}, \sigma_{c_{2}}$, and $\sigma_{c_{3}}$. At time $t+n+1$, since neurons $\sigma_{c_{1}}$ and $\sigma_{c_{2}}$ each have two spikes, the spikes are removed by rule $\left(a^{2}, \bar{a}\right) / a^{2} \rightarrow \lambda$. Moreover, neuron $\sigma_{c_{3}}$ sends two spikes to neuron $\sigma_{l_{0}}$. Consequently, from time $t+2$ to time $t+n+1$, neuron $\sigma_{1}$ contains $2 n$ spikes in total (i.e., the number of spikes in register 1 is $n$ ), and due to two spikes in neuron $\sigma_{l_{0}}$, the system starts to simulate the initial instruction, $l_{0}$.

In the case of accepting mode, deterministic ADD instructions, of the form $l_{i}:\left(A D D(r), l_{j}\right)$, are used in the register machine, as shown in Fig. 7. Note that there is no inhibitory neuron in this module. Suppose that two spikes are received by neuron $\sigma_{l_{i}}$ at time $t$. At time $t+1$, due to two spikes in neuron $\sigma_{l_{i}}$, rule $a^{2} \leftarrow a^{2}$ is applied to send two spikes to neurons $\sigma_{l_{j}}$ and $\sigma_{r}$. Hence neuron $\sigma_{r}$ has two spikes, meaning that register $r$ is incremented by 1 . With two spikes in neuron $\sigma_{l_{j}}$, the system starts to simulate instruction $l_{j}$.

Module SUB remains unchanged, as shown in Fig. 4. Module FIN is ignored, but neuron $\sigma_{l_{h}}$ remains in the system. When neuron $\sigma_{l_{h}}$ has two spikes, this indicates that halting instruction $l_{h}$ is reached, and register machine $M$ stops.

From the discussion above, the SNP-IR system correctly simulates the register machine working in accepting mode, where each neuron contains two rules at most. Therefore, the theorem holds.

\subsection{SNP-IR systems as function computing devices}

A small universal SNP-IR system will be constructed to compute functions. The register machine $M=\left(m, H, l_{0}, l_{h}, I\right)$ used to compute the function $f: N^{k} \rightarrow N$ can be illustrated as follows. Initially, $k$ arguments are introduced in $k$ special registers (usually, the first $k$ registers are used), and all of the registers are assumed to be empty. The machine starts from instruction $l_{0}$, and it executes constantly until the halting instruction $l_{h}$ is reached. At this moment, the function value of $f$ is the number stored in another special register, $r_{t}$. Denote by $\left(\varphi_{0}, \varphi_{1}, \ldots\right)$ a fixed admissible enumeration of the unary partial recursive functions. 


$$
\begin{array}{lll}
l_{0}:\left(S U B(1), l_{1}, l_{2}\right) & l_{1}:\left(A D D(7), l_{0}\right) & l_{2}:\left(A D D(6), l_{3}\right) \\
l_{3}:\left(S U B(5), l_{2}, l_{4}\right) & l_{4}:\left(S U B(6), l_{5}, l_{3}\right) & l_{5}:\left(A D D(5), l_{6}\right) \\
l_{6}:\left(S U B(7), l_{7}, l_{8}\right) & l_{7}:\left(A D D(1), l_{4}\right) & l_{8}:\left(S U B(6), l_{9}, l_{0}\right) \\
l_{9}:\left(A D D(6), l_{10}\right) & l_{10}:\left(S U B(4), l_{0}, l_{11}\right) & l_{11}:\left(S U B(5), l_{12}, l_{13}\right) \\
l_{12}:\left(S U B(5), l_{14}, l_{15}\right) & \left.l_{13}:\left(S U B(2), l_{18}\right\}, l_{19}\right) & l_{14}:\left(S U B(5), l_{16}, l_{17}\right) \\
l_{15}:\left(S U B(3), l_{18}, l_{20}\right) & l_{16}:\left(A D D(4), l_{11}\right) & l_{17}:\left(A D D(2), l_{21}\right) \\
l_{18}:\left(\operatorname{SUB}(4), l_{0}, l_{22}\right) & l_{19}:\left(S U B(0), l_{0}, l_{18}\right) & l_{20}:\left(A D D(0), l_{0}\right) \\
l_{21}:\left(A D D(3), l_{18}\right) & l_{22}:\left(\operatorname{SUB}(0), l_{23}, l_{24}\right) & l_{23}:\left(A D D(8), l_{22}\right) \\
l_{24}: \operatorname{HALT} & &
\end{array}
$$

Fig. 8. The small universal register machine $M_{u}^{\prime}$.

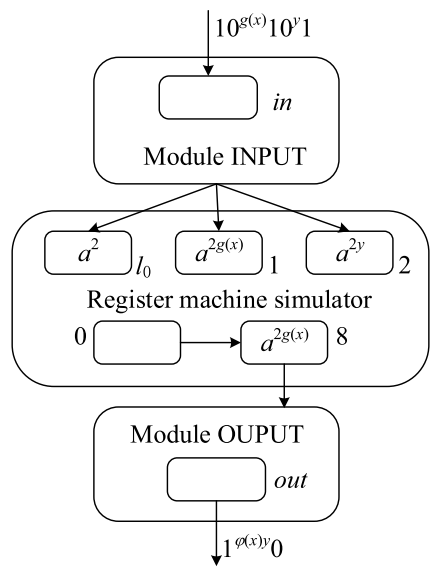

Fig. 9. General design of universal SNP-IR system $\Pi_{3}$.

A register machine is said to be universal only if there exists a recursive function $g$ such that $\varphi_{x}(y)=M_{u}(g(x), y)$ holds for all natural numbers $x, y$.

Korec [67] introduced a well-known small universal register machine for computing functions: $M_{u}=\left(8, H, l_{0}, l_{h}, I\right)$. The register machine $M_{u}$ has 23 instructions and 8 registers (labeled 0 through 7). By importing two numbers $g(x)$ and $y$ in registers 1 and 2, respectively, any $\varphi_{x}(y)$ can be computed by the register machine $M_{u}$; when the machine halts, the function value is stored in register 0 . An SNP-IR system will be designed to simulate the register machine $M_{u}$. For simplicity, we modify the register machine $M_{u}$ by adding a new register 8 and replacing the original halting instruction by $l_{22}:\left(\operatorname{SUB}(0), l_{23}, l_{h}\right), l_{23}$ : $\left(A D D(8), l_{22}\right), l_{h}: H A L T$. Denote by $M_{u}^{\prime}$ the modification of $M_{u}$, as shown in Fig. 8. Therefore, register machine $M_{u}^{\prime}$ contains 24 ADD and SUB instructions, 9 registers, and 25 labels.

Theorem 3. There exists a small universal SNP-IR system having 100 neurons for computing functions.

Proof. We design an SNP-IR system $\Pi_{3}$ to simulate the universal register machine $M_{u}^{\prime}$. The SNP-IR system $\Pi_{3}$ includes an INPUT module, an OUTPUT module, and several ADD and SUB modules to simulate the ADD and SUB instructions, respectively, of $M_{u}^{\prime}$. The INPUT module is used to import a spike train from the environment, and the OUTPUT module exports the computational result.

Fig. 9 shows the general design of the universal SNP-IR system $\Pi_{3}$. Each register $r$ in $M_{u}^{\prime}$ corresponds to a neuron $\sigma_{r}$, and if register $r$ stores the number $n \geq 0$, then neuron $\sigma_{r}$ has $2 n$ spikes. Moreover, neuron $\sigma_{l_{i}}$ in $\Pi_{3}$ corresponds to instruction $l_{i}$

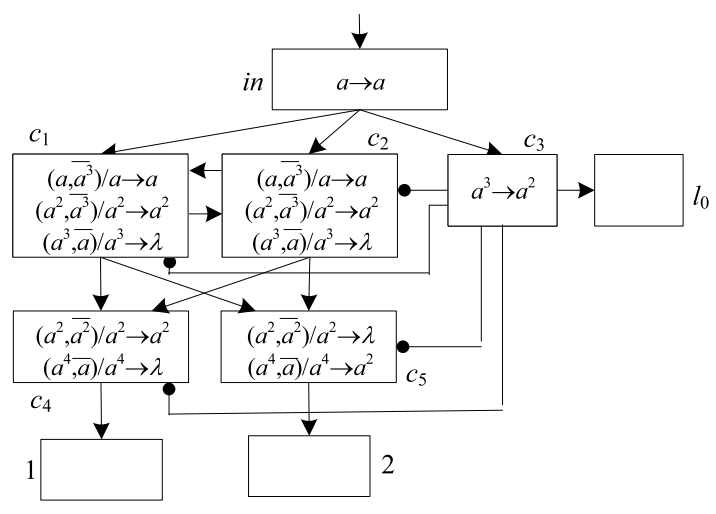

Fig. 10. INPUT module.

in $M_{u}^{\prime}$. When neuron $\sigma_{l_{i}}$ receives two spikes, it starts to simulate the instruction $l_{i}$. Once neuron $\sigma_{l_{h}}$ receives two spikes, $M_{u}^{\prime}$ is completely simulated by the system $\Pi_{3}$. Finally, the first two spikes sent to the environment by the output neuron $\sigma_{\text {out }}$ are regarded as the computational result (stored in register 8 ). In the initial configuration, assume that all of the neurons are empty.

Fig. 10 shows the INPUT module, which is used to read the spike train $10^{g(x)} 10^{y} 1$ from the environment, where $2 g(x)$ spikes are stored in neuron $\sigma_{1}$ and $2 y$ spikes are placed in neuron $\sigma_{2}$.

Assume that at time $t_{1}$, neuron $\sigma_{\text {in }}$ receives the first spike from the environment. Similar to the analysis of the INPUT module in the proof of Theorem 2, at time $t_{1}+1$, rule $a \rightarrow a$ in neuron $\sigma_{\text {in }}$ is applied to send a spike to neurons $\sigma_{c_{1}}, \sigma_{c_{2}}$, and $\sigma_{c_{3}}$. Note that neuron $\sigma_{c_{3}}$ is the inhibitory neuron of neurons $\sigma_{c_{2}}$ and $\sigma_{c_{1}}$. At time $t_{2}+2$, because neuron $\sigma_{c_{3}}$ contains only a spike, rule $\left(a, \overline{a^{3}}\right) / a \rightarrow a$ is enabled in neurons $\sigma_{c_{1}}$ and $\sigma_{c_{2}}$ and applied to send a spike to neurons $\sigma_{c_{4}}$ and $\sigma_{c_{5}}$, and they exchange a spike with each other. Note that neuron $\sigma_{c_{3}}$ is also the inhibitory neuron of neurons $\sigma_{c_{4}}$ and $\sigma_{c_{5}}$. At time $t_{2}+3$, with a spike in neuron $\sigma_{c_{3}}$, rule $\left(a^{2}, \overline{a^{2}}\right) / a^{2} \rightarrow a^{2}$ in neuron $\sigma_{c_{4}}$ is enabled, but neuron $\sigma_{c_{5}}$ is inhibited. Neuron $\sigma_{c_{4}}$ sends two spikes to neuron $\sigma_{1}$, and two spikes in neuron $\sigma_{c_{5}}$ are consumed by rule $\left(a^{2}, \overline{a^{2}}\right) / a^{2} \rightarrow \lambda$. The process is repeated until the second spike arrives at neurons $\sigma_{c_{1}}$, $\sigma_{c_{2}}$, and $\sigma_{c_{3}}$. During each step, two spikes in neuron $\sigma_{c_{4}}$ are added to neuron $\sigma_{1}$. Therefore, from time $t_{1}+3$ to time $t_{1}+g(x)+2$, neuron $\sigma_{1}$ receives in total $2 g(x)$ spikes (i.e., the number of spikes in register 1 is $g(x)$ ).

Assume that neuron $\sigma_{\text {in }}$ receives the second spike at time $t_{2}$ (in fact, $t_{2}=t_{1}+g(x)+2$ ). Similarly, at time $t_{2}+1$, rule $a \rightarrow a$ in neuron $\sigma_{i n}$ is applied to send a spike to neurons $\sigma_{c_{1}}$, $\sigma_{c_{2}}$, and $\sigma_{c_{3}}$. At this time, neurons $\sigma_{c_{1}}, \sigma_{c_{2}}$, and $\sigma_{c_{3}}$ each have two spikes. At time $t_{1}+2$, since neuron $\sigma_{c_{3}}$ has two spikes, rule 


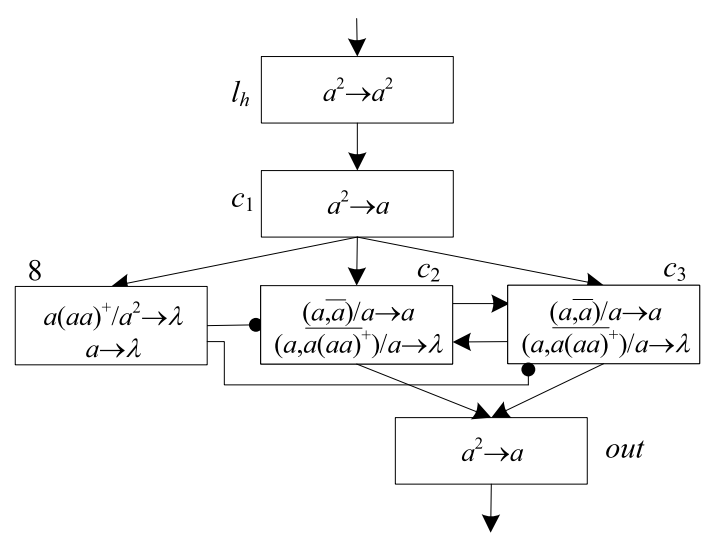

Fig. 11. OUTPUT module.

$\left(a^{2}, \overline{a^{3}}\right) / a^{2} \rightarrow a^{2}$ is enabled in neurons $\sigma_{c_{1}}$ and $\sigma_{c_{2}}$ and applied to send two spikes to neurons $\sigma_{c_{4}}$ and $\sigma_{c_{5}}$, and they exchange two spikes with each other. At time $t_{1}+3$, due to two spikes in neuron $\sigma_{c_{3}}$, rule $\left(a^{4}, \bar{a}\right) / a^{4} \rightarrow a^{2}$ in neuron $\sigma_{c_{5}}$ is enabled, but neuron $\sigma_{c_{4}}$ is inhibited. Neuron $\sigma_{c_{5}}$ sends two spikes to neuron $\sigma_{2}$, and four spikes in neuron $\sigma_{c_{5}}$ are consumed by rule $\left(a^{4}, \overline{a^{2}}\right) / a^{4} \rightarrow \lambda$. The process is repeated until the third spike arrives at neurons $\sigma_{c_{1}}, \sigma_{c_{2}}$, and $\sigma_{c_{3}}$. During each step, two spikes in neuron $\sigma_{c_{5}}$ are constantly added to neuron $\sigma_{2}$. Hence, from time $t_{2}+3$ to time $t_{2}+y+2$, neuron $\sigma_{2}$ receives in total $2 y$ spikes (i.e., the number of spikes in register 2 is $y$ ). Since neuron $\sigma_{3}$ has three spikes, it fires to send two spikes to neuron $\sigma_{l_{0}}$, meaning that the system starts to simulate the initial instruction $l_{0}$.

From Fig. 8, we can observe that all of the ADD instructions have the form $l_{i}:\left(A D D(r), l_{j}\right)$. Consequently, a deterministic ADD module, whose working principle was discussed in the proof of Theorem 2, can be used in the simulation of the ADD instruction, as shown in Fig. 7.

The SUB module in Fig. 4 is used in the simulation of SUB instruction $l_{i}$ : $\left(S U B(r), l_{j}, l_{k}\right)$. The working principle of the SUB module was described in the proof of Theorem 1 .

Suppose that $M$ halts now, i.e., the instruction $l_{h}$ is reached. The computational result is contained in register 8 , and it never decreases during the computation. The computational result is exported by an OUTPUT module, as shown in Fig. 11.

Assume that neuron $\sigma_{l_{h}}$ has two spikes at time $t$, meaning that $M$ halts, and neuron $\sigma_{8}$ contains $2 n$ spikes (indicating the number $n$ in register 8). At time $t+1$, neuron $\sigma_{l_{h}}$ sends two spikes to neuron $\sigma_{c_{1}}$. At time $t+2$, neuron $\sigma_{c_{1}}$ sends a spike to neurons $\sigma_{8}, \sigma_{c_{2}}$, and $\sigma_{c_{3}}$. Thus neuron $\sigma_{8}$ receives a spike, and it contains an odd number of spikes. Note that neuron $\sigma_{8}$ is the inhibitory neuron of neurons $\sigma_{c_{2}}$ and $\sigma_{c_{3}}$. At time $t+3$, rule $(a, \bar{a}) / a \leftarrow a$ in neurons $\sigma_{c_{2}}$ and $\sigma_{c_{3}}$ is applied to send a spike to neuron $\sigma_{\text {out }}$, and they exchange a spike with each other. At this time, two spikes are consumed in neuron $\sigma_{8}$ (i.e., the number in register 8 is decremented by 1 ). At time $t+4$, neuron $\sigma_{\text {out }}$ sends the first spike to the environment. The process is repeated until only one spike is stored in neuron $\sigma_{8}$, and neuron $\sigma_{\text {out }}$ sends a spike to the environment each time. At time $t+n+3$, because only one spike is in neuron $\sigma_{8}$, and neurons $\sigma_{c_{2}}$ and $\sigma_{c_{2}}$ each have a spike, rule $\left(a, \overline{a(a a)^{+}}\right) / a \leftarrow \lambda$ is enabled. Thus the spike in neurons $\sigma_{c_{2}}$ and $\sigma_{c_{2}}$ is consumed. Consequently, from step $t+4$ to step $t+n+3$, neuron $\sigma_{\text {out }}$ sends in total $n$ spikes to the environment, which is exactly the number contained in register 8 when $M$ halts.

From the discussion above, the system $\Pi_{3}$ correctly simulates the register machine $M_{u}^{\prime}$. In SNP-IR system $\Pi_{3}$, we use a total of 100 neurons: (i) six neurons for the INPUT module; (ii) four
Table 1

Comparison of different computing models in terms of small numbers of computing units.

\begin{tabular}{ll} 
Computing models & Number of neurons \\
\hline SNP-IR systems & 100 \\
PSNP systems [38] & 200 \\
SNQ P systems with one type of spike [40] & 181 \\
Recurrent neural networks [68] & 886 \\
\hline
\end{tabular}

neurons for the OUTPUT module; (iii) 56 auxiliary neurons for 14 SUB instructions; (iv) nine neurons for nine registers; and (v) 25 neurons for 25 instructions.

Theorem 3 shows a small number of computing units (i.e., neurons) for SNP-IR systems as function-computing devices to achieve Turing universality. To further evaluate the computational power of SNP-IR systems, Table 1 compares the proposed variant with other computing models in terms of small numbers of computing units. From Table 1, we can observe that recurrent neural networks [68], PSNP systems [38], and SNQ P systems with one type of spike [40] need 886, 200, and 181 neurons, respectively, to achieve Turing universality for the computing function, and SNP-IR systems need fewer neurons than all of these.

\section{Conclusions and further work}

We have proposed a new model of SNP systems, SNP-IR systems, which introduce inhibitory rules. These are inspired by the mechanism of inhibitory synapses, whereas usual firing rules are related to the mechanism of the excitatory synapse. Different from usual firing rules, the firing condition of an inhibitory rule not only depends on the state of the neuron where the rule resides, but also is related to the states of its inhibitory neurons. Therefore, whether a neuron fires in SNP-IR systems may be controlled by other neurons, i.e., its inhibitory neurons. This interesting feature, which SNP systems lack, indicates that SNP-IR systems have a stronger control ability. From the perspective of a directed graph, if neuron $\sigma_{j}$ is an inhibitory neuron of neuron $\sigma_{i}$, then there exists a special arc from neuron $\sigma_{j}$ to neuron $\sigma_{i}$, called an inhibitory arc. Different from usual arcs, inhibitory arcs do not transmit spikes; they only play the role of controlling a neuron's firing. The computational power of SNP-IR systems was investigated. The universality of SNP-IR systems as number-accepting/generating devices was proved. Moreover, we established a small universality result of SNP-IR systems for computing functions: a small universal function-computing device consisting of 100 neurons was constructed.

As stated in the existing SNP systems, some problems that refer to SNP-IR systems will be discussed, such as language generator, and asynchronous and sequential modes. Moreover, it is worth studying how to integrate other mechanisms or strategies in SNP-IR systems, so as to propose more models.

As stated above, since inhibitory rule of the form $\left(E_{e n}, \overline{E_{i n}}\right) / a^{c}$ $\rightarrow a^{p}$ is introduced SNP-IR systems, the firing of a neuron is controlled by both its own state and the states of its inhibitory neurons. However, the firing of a neuron in the existing SNP systems is controlled only by its own state. Therefore, SNP-IR systems can provide a stronger control ability than the existing SNP systems. This is a new and interesting attribute, which makes them more suitable for dealing with some practical application problems, for example, supervisory control problems in discrete event systems. The goal of supervisory control is to restrict the behavior of a system to satisfy the desired control specifications, such as deadlock avoidance or liveness enforcement in discrete event system. However, when the existing SNP systems are used 
to describe the discrete event system, they may suffer from the state explosion problem. Due to inhibitory rules, SNP-IR systems have a potential advantage to simplify the structure of model and reduce the corresponding state space so as to achieve the optimal supervisory control. Therefore, future work will address the application of SNP-IR systems in supervisory control problems in discrete event systems.

\section{Acknowledgments}

The authors thank the anonymous reviewers for providing very insightful and constructive suggestions, which have greatly help improve the presentation of this paper.

\section{References}

[1] G. Păun, Computing with membranes, J. Comput. Syst. Sci. 61 (1) (2000) 108-143, http://dx.doi.org/10.1006/jcss.1999.1693.

[2] G. Paun, G. Rozenberg, A. Salomaa, The Oxford Handbook of Membrane Computing, Oxford University Press, Inc., 2010.

[3] R. Freund, G. Păun, M. Pérez-Jiménez, Tissue-like P systems with channelstates, Theoret. Comput. Sci. 330 (1) (2005) 101-116, http://dx.doi.org/10. 1016/j.tcs.2004.09.013.

[4] F. Bernardini, M. Gheorghe, Population P systems, J. UCS 10 (5) (2004) 509-539, http://dx.doi.org/10.3217/jucs-010-05-0509.

[5] G. Păun, R. Păun, Membrane computing and economics: numerical $P$ systems, Fund. Inform. 73 (1,2) (2006) 213-227, http://dx.doi.org/10.3217/ jucs-010-05-0509.

[6] L. Ciencialová, E. Csuhaj-Varjú, A. Kelemenová, G. Vaszil, Variants of P colonies with very simple cell structure, Int. J. Comput. Commun. Control IV (3) (2009) 224-233, http://dx.doi.org/10.15837/ijccc.2009.3.2430.

[7] A. Spicher, O. Michel, M. Cieslak, J. Giavitto, P. Prusinkiewicz, Stochastic $\mathrm{P}$ systems and the simulation of biochemical processes with dynamic compartments, BioSystems 91 (3) (2008) 458-472, http://dx.doi.org/10. 1016/j.biosystems.2006.12.009.

[8] M. Gheorghe, F. Ipate, R. Lefticaru, M.J. Pérez-Jiménez, A. Turcanu, L. Valencia-Cabrera, M. García-Quismondo, L. Mierlă, 3-col problem modelling using simple kernel P systems, Int. J. Comput. Math. 90 (4) (2013) 816-830, http://dx.doi.org/10.1080/00207160.2012.743712.

[9] G. Păun, M. Pérez-Jiménez, Solving problems in a distributed way in membrane computing: dP systems, Int. J. Comput. Commun. Control V (2) (2010) 238-250, http://dx.doi.org/10.1080/00207160.2012.743712.

[10] B. Song, C. Zhang, L. Pan, Tissue-like P systems with evolutional symport/antiport rules, Inform. Sci. 378 (C) (2016) 177-193, http://dx.doi.org/ 10.1016/j.ins.2016.10.046.

[11] Z. Zhang, T. Wu, A. Pǎun, L. Pan, Numerical P systems with migrating variables, Theoret. Comput. Sci. 641 (C) (2016) 85-108, http://dx.doi.org/ 10.1016/j.tcs.2016.06.004.

[12] G. Ciobanu, G. Păun, M. Pérez-Jiménez, Applications of Membrane Computing, Berlin: Springer-Verlag, 2006.

[13] G. Păun, M. Pérez-Jiménez, Membrane computing: brief introduction, recent results and applications, BioSystems 85 (1) (2006) 11-22, http: //dx.doi.org/10.1016/j.biosystems.2006.02.001.

[14] Y. Zhao, X. Liu, J. Qu, The k-medoids clustering algorithm by a class of P system, J. Inf. Comput. Sci. 9 (18) (2012) 5777-5790.

[15] H. Peng, J. Wang, M. Pérez-Jiménez, A. Riscos-Núñez, An unsupervised learning algorithm for membrane computing, Inform. Sci. 304 (20) (2015) 80-91, http://dx.doi.org/10.1016/j.ins.2015.01.019.

[16] H. Peng, J. Wang, P. Shi, M. Pérez-Jiménez, A. Riscos-Núñez, An extended membrane system with active membrane to solve automatic fuzzy clustering problems, Int. J. Neural Syst. 26 (3) (2016) 1-17, http://dx.doi.org 10.1142/S0129065716500040.

[17] H. Peng, P. Shi, J. Wang, A. Riscos-Núñez, M. Pérez-Jiménez, Multiobjective fuzzy clustering approach based on tissue-like membrane systems, Knowl.Based Syst. 125 (2017) 74-82, http://dx.doi.org/10.1016/j.knosys.2017.03. 024.

[18] D. Díaz-Pernil, A. Berciano, F. Peña-Cantillana, M. Gutiérrez-Naranjo, Segmenting images with gradient-based edge detection using membrane computing, Pattern Recognit. Lett. 34 (8) (2013) 846-855, http://dx.doi. org/10.1016/j.knosys.2017.03.024.

[19] H. Peng, J. Wang, M. Pérez-Jiménez, Optimal multi-level thresholding with membrane computing, Digit. Signal Process. 37 (2015) 53-64, http://dx. doi.org/10.1016/j.dsp.2014.10.006.

[20] H. Peng, J. Wang, M. Pérez-Jiménez, P. Shi, A novel image thresholding method based on membrane computing and fuzzy entropy, J. Intell. Fuzzy Systems 24 (2) (2013) 229-237, http://dx.doi.org/10.3233/IFS-2012-0549.
[21] R.I. Yahya, S. Hasan, L. George, B. Alsalibi, Membrane computing for 2D image segmentation, Int. J. Adv. Soft Comput. Appl. 7 (1) (2015) 35-50.

[22] G. Zhang, M. Gheorghe, Y. Li, A membrane algorithm with quantuminspired subalgorithms and its application to image processing, Nat. Comput. 11 (4) (2012) 701-717, http://dx.doi.org/10.1007/s11047-0129320-2.

[23] H. Peng, J. Wang, M.J. Pérez-Jiménez, A. Riscos-Núñez, The framework of P systems applied to solve optimal watermarking problem, Signal Process. 101 (2014) 256-265, http://dx.doi.org/10.1016/j.sigpro.2014.02.020.

[24] J. Wang, P. Shi, H. Peng, Membrane computing model for IIR filter design, Inform. Sci. 329 (2016) 164-176, http://dx.doi.org/10.1016/j.ins.2015.09. 011.

[25] C. Buiu, C. Vasile, O. Arsene, Development of membrane controllers for mobile robots, Inform. Sci. 187 (2012) 33-51, http://dx.doi.org/10.1016/j. ins.2011.10.007.

[26] X. Wang, G. Zhang, F. Neri, T. Jiang, J. Zhao, M. Gheorghe, F. Ipate, R. Lefticaru, Design and implementation of membrane controllers for trajectory tracking of nonholonomic wheeled mobile robots, Integr. Comput.-Aided Eng. 23 (1) (2016) 15-30, http://dx.doi.org/10.3233/ICA-150503.

[27] M. Gheorghe, V. Manca, F. Romero-Campero, Deterministic and stochastic P systems for modelling cellular processes, Nat. Comput. 9 (2) (2010) 457-473.

[28] M. García-Quismondo, M. Levin, D. Lobo-Fernández, Modeling regenerative processes with membrane computing, Inform. Sci. 381 (2017) 229-249, http://dx.doi.org/10.1016/j.ins.2016.11.017.

[29] M. García-Quismondo, I. Nisbet, C. Mostello, M. Reed, Modeling population dynamics of roseate terns (sterna dougallii) in the northwest atlantic ocean, Ecol. Model. 68 (2018) 298-311, http://dx.doi.org/10.1016/ j.ecolmodel.2017.12.007.

[30] M. Ionescu, G. Păun, T. Yokomori, Spiking neural P systems, Fundam. Inform. 71 (2-3) (2006) 279-308.

[31] G. Păun, Spiking neural P systems with astrocyte-like control, J. UCS 13 (11) (2007) 1707-1721, http://dx.doi.org/10.3217/jucs-013-11- 1707.

[32] L. Pan, J. Wang, H. Hoogeboom, Spiking neural P systems with astrocytes, Neural Comput. 24 (3) (2012) 805-825, http://dx.doi.org/10.1162/NECO_a_ 00238.

[33] L. Pan, G. Păun, Spiking neural P systems with anti-spikes, Int. J. Comput. Commun. Control IV (3) (2009) 273-282, http://dx.doi.org/10.15837/ijccc. 2009.3.2435.

[34] H. Peng, J. Yang, J. Wang, T. Wang, Z. Sun, X. Song, X. Luo, X. Huang, Spiking neural P systems with multiple channels, Neural Netw. 95 (2017) 66-71, http://dx.doi.org/10.1016/j.neunet.2017.08.003.

[35] T. Song, L. Pan, G. Păun, Spiking neural P systems with rules on synapses, Theoret. Comput. Sci. 529 (2014) 82-95, http://dx.doi.org/10.1016/j.tcs. 2014.01.001.

[36] H. Peng, R. Chen, J. Wang, X. Song, T. Wang, F. Yang, Z. Sun, Competitive spiking neural P systems with rules on synapses, IEEE Trans. NanoBiosci. 16 (8) (2018) 888-895, http://dx.doi.org/10.1109/TNB.2017.2783890.

[37] H. Chen, T.-O. Ishdorj, G. Păun, Computing along the axon, Prog. Nat. Sci. 17 (4) (2007) 417-423.

[38] T. Wu, A. Păun, Z. Zhang, L. Pan, Spiking neural P systems with polarizations, IEEE Trans. Neural Netw. Learn. Syst. 29 (8) (2018) 3349-3360, http://dx.doi.org/10.1109/TNNLS.2017.2726119.

[39] F. Cabarle, H. Adorna, M. Jiang, X. Zeng, Spiking neural P systems with scheduled synapses, IEEE Trans. Nanobiosci. 27 (5) (2016) 1337-1347, http://dx.doi.org/10.1109/TNB.2017.2762580.

[40] L. Pan, G. Păun, G. Zhang, F. Neri, Spiking neural P systems with communication on request, Int. J. Neural Syst. 28 (8) (2017) 1-13, http: //dx.doi.org/10.1142/S0129065717500423.

[41] O. Ibarra, A. Păun, A. Rodríguez-Paton, Sequential SNP systems based on $\mathrm{min} / \mathrm{max}$ spike number, Theoret. Comput. Sci. 410 (30) (2009) 2982-2991, http://dx.doi.org/10.1016/j.tcs.2009.03.004.

[42] X. Zhang, X. Zeng, B. Luo, L. Pan, On some classes of sequential spiking neural P systems, Neural Comput. 26 (5) (2014) 974-997, http://dx.doi. org/10.1162/NECO_a_00580.

[43] J. Wang, H. Hoogeboom, L. Pan, G. Păun, M. Pérez-Jiménez, Spiking neural P systems with weights, Neural Comput. 22 (2010) 2615-2646, http: //dx.doi.org/10.1162/NECO_a_00022.

[44] H. Peng, J. Wang, M. Pérenz-Jiménez, A. Riscos-Núñez, Dynamic threshold neural P systems, Knowl.-Based Syst. 163 (2019) 875-884, http://dx.doi. org/10.1016/j.knosys.2018.10.016.

[45] H. Peng, J. Wang, Coupled neural P systems, IEEE Trans. Neural Netw. Learn. Syst. (2018) http://dx.doi.org/10.1109/TNNLS.2018.2871999.

[46] X. Zeng, X. Zhang, T. Song, L. Pan, Spiking neural P systems with thresholds, Neural Comput. 26 (7) (2014) 1340-1361, http://dx.doi.org/10.1162/NECO_ a_00605.

[47] M. Cavaliere, O. Ibarra, G. Păun, O. Egecioglu, M. Ionescu, S. Woodworth, Asynchronous spiking neural P systems, Theoret. Comput. Sci. 410 (24) (2009) 2352-2364. 
[48] T. Song, L. Pan, G. Păun, Asynchronous spiking neural P systems with local synchronization, Inform. Sci. 219 (2012) 197-207, http://dx.doi.org/ 10.1016/j.ins.2012.07.023.

[49] H. Peng, J. Wang, M. Pérez-Jiménez, H. Wang, J. Shao, T. Wang, Fuzzy Reasoning spiking neural $P$ system for fault diagnosis, Inform. Sci. 235 (2013) 106-116, http://dx.doi.org/10.1016/j.ins.2012.07.015.

[50] J. Wang, P. Shi, H. Peng, M. Pérez-Jiménez, T. Wang, Weighted fuzzy spiking neural P systems, IEEE Trans. Fuzzy Syst. 21 (2) (2013) 209-220, http://dx.doi.org/10.1109/TFUZZ.2012.2208974.

[51] J. Wang, H. Peng, W. Yu, J. Ming, M. Pérenz-Jiménez, C. Tao, X. Huang, Interval-valued fuzzy spiking neural $\mathrm{P}$ systems for fault diagnosis of power transmission networks, Eng. Appl. Artif. Intell. 82 (2019) 102-109, http: //dx.doi.org/10.1016/j.engappai.2019.03.014.

[52] H. Chen, R. Freund, M. Ionescu, G. Păun, M. Pérez-Jiménez, On string languages generated by spiking neural P systems, Fund. Inform. 75 (1) (2007) 141-162.

[53] X. Zhang, X. Zeng, L. Pan, On string language generated by spiking neural P systems with exhaustive use of rules, Nat. Comput. 90 (1) (2008) 535-549, http://dx.doi.org/10.1007/s11047-008-9079-7.

[54] A. Păun, G. Păun, Small universal spiking neural P systems, BioSystems 90 (1) (2007) 48-60, http://dx.doi.org/10.1016/j.biosystems.2006.06.006.

[55] A. Păun, M. Sidoroff, Sequentially induced by spike number in SNP systems: small universal machines, in: Membrane Computing, Springer, 2012, pp. 333-345.

[56] G. Xiong, D. Shi, L. Zhu, X. Duan, A new approach to fault diagnosis of power systems using fuzzy reasoning spiking neural $P$ systems, Math. Probl. Eng. 2013 (1) (2013) 211-244, http://dx.doi.org/10.1155/2013/ 815352.

[57] T. Wang, G. Zhang, J. Zhao, Z. He, J. Wang, M. Pérez-Jiménez, Fault diagnosis of electric power systems based on fuzzy reasoning spiking neural P systems, IEEE Trans. Power Syst. 30 (3) (2015) 1182-1194, http: //dx.doi.org/10.1109/TPWRS.2014.2347699.
[58] H. Peng, J. Wang, P. Shi, M. Pérez-Jiménez, A. Riscos-Núñez, Fault diagnosis of power systems using fuzzy tissue-like P systems, Integr. Comput.-Aided Eng. 24 (4) (2017) 401-411, http://dx.doi.org/10.3233/ICA-170552.

[59] H. Peng, J. Wang, J. Ming, P. Shi, M.J. Pérez-Jiménez, W. Yu, C. Tao, Fault diagnosis of power systems using intuitionistic fuzzy spiking neural $P$ systems, IEEE Trans. Smart Grid (2017) 1-8, http://dx.doi.org/10.1109/TSG. 2017.2670602.

[60] D. Díaz-Pernil, F. Peña-Cantillana, M. Gutiérrez-Naranjo, A parallel algorithm for skeletonizing images by using spiking neural $\mathrm{P}$ systems, Neurocomputing 115 (2013) 81-91, http://dx.doi.org/10.1016/j.neucom. 2012.12.032.

[61] G. Zhang, H. Rong, F. Neri, M. Pérez-Jiménez, An optimization spiking neural $\mathrm{P}$ system for approximately solving combinatorial optimization problems, Int. J. Neural Syst. 24 (05) (2014) 1-16.

[62] J. Nicholls, A. Martin, P. Fuchs, D. Brown, M. Diamond, D. Weisblat, From Neuron to Brain, fifth ed., Sinuer Assocites, 2012.

[63] E. Kandel, J. Schwartz, T. Jessell, S. Siegelbaum, A. Hudspeth, Principles of Neural Science, fifth ed., McGraw-Hill, 2015.

[64] M. Sheng, C. Hoogenraad, Hoogenraad, The postsynaptic architecture of excitatory synapses: a more quantitative view, Annu. Rev. Biochem. 76 (1) (2007) 823-847, http://dx.doi.org/10.1146/annurev.biochem.76.060805 160029.

[65] C. Jean-Xavier, J. Pflieger, S. Liabeuf, L. Vinay, Inhibitory postsynaptic potentials in lumbar motoneurons remain depolarizing after neonatal spinal cord transection in the rat, J. Neurophysiol. 96 (5) (2006) 2274-2281, http://dx.doi.org/10.1152/jn.00328.2006.

[66] G. Rozenberg, A. Salomaa, Handbook of Formal Languages: Beyonds words, Springer Science \& Business Media, 1997.

[67] I. Korec, Small universal register machines, Theoret. Comput. Sci. 168 (2) (1996) 267-301, http://dx.doi.org/10.1016/S0304-3975(96)00080-1.

[68] H. Siegelmann, E. Sontag, On the computational power of neural nets, J Comput. System Sci. 50 (1) (1995) 132-150. 